General Mathematics Vol. 28, No. 2 (2020), 87-96

DOI: $10.2478 / \mathrm{gm}-2020-0018$

S sciendo

\title{
Geometric properties of some applications of the Tremblay operator ${ }^{1}$
}

\author{
Hüseyin Irmak
}

\begin{abstract}
By this research notes, the well-known Tremblay operator and certain core knowledge therewith are firstly introduced and an extensive result containing numerous (analytic and) geometric properties (of possible applications of the related operator) along with a number of special implications are then constituted. As method for proving, the well-known assertion proposed by [8] is also considered there.
\end{abstract}

2010 Mathematics Subject Classification: 26A33, 30C45, 35A23, $26 \mathrm{D} 10$.

Key words and phrases: complex plane, open unit disc, analytic functions, operator of fractional order type, Tremblay operator in the complex plane.

\section{Introduction and preliminaries}

As is sufficiently presented in the works in the references of this investigation, the well-known fractional calculus, which is fractional integral(s) and fractional derivative(s), as we know, is a generalization of the classical calculus to pertinent to operations of integration and also differentiation of integer, non-integer or fractional order. Nevertheless, the concept of the operators of fractional order has been described almost concurrently with the development of the classical ones. Since the 19th century, this theory and also its implementations have rapidly improved.

The literature also shows that there are a great number of works relating to both theoretical results and their practical applications. In particular, by the help of new definitions, there also are several results associating with several relations between certain results specified by fractional calculus (or many operators in certain differential forms). By this present research, as certain extra operators constituted

\footnotetext{
${ }^{1}$ Received 14 February, 2020

Accepted for publication (in revised form) 19 October, 2020
} 
by fractional calculus, one of those will be firstly identified and some (of geometric properties of their) implementations will be also exhibited. For those, there will be needed to recall certain information and also definitions are directly connected to certain applications of the Tremblay operator stated by fractional calculus. In special, for possible implementations of the Tremblay operator, the recent papers given in [3] and [5]-[7] can be given as examples.

Now, we begin by introducing both the related operators and certain information which will be required for this research.

Firstly, let the well-known notations $\mathbb{N}, \mathbb{R}, \mathbb{C}$ and $\mathbb{U}$ be the set of positive integers, be the set of real numbers, be the set of complex numbers, and be the well-known open unit disc $\mathbb{U}:=\{z \in \mathbb{C}:|z|<1\}$, respectively.

Let the notations:

$$
\mathrm{A}(n) \text { and } \mathrm{H}_{e}(n)
$$

denote the families of the complex valued functions $g(z)$ and $h(z)$ by the TaylorMaclaurin series being of the forms:

$$
g(z)=z+b_{n+1} z^{n+1}+b_{n+2} z^{n+2}+\cdots \quad(n \in \mathbb{N})
$$

and

$$
h(z)=e+e_{n} z^{n}+e_{n+1} z^{n+1}+\cdots \quad(n \in \mathbb{N}),
$$

which are analytic (and univalent) in the open disc $\mathbb{U}$, respectively. In particular, also let $\mathrm{H}(n):=\mathrm{H}_{1}(n)$.

Let $\omega:=\omega(z)$ be an analytic function in a simply-connected region of the complex plane containing the origin. The definition of fractional derivative of function $\omega$ is generally denoted by the symbol:

$$
\mathrm{D}_{z}^{\lambda}[\omega](z) \quad(0 \leq \lambda<1)
$$

and its fractional derivative of order $\lambda$ is then defined by

$$
\mathrm{D}_{z}^{\lambda}[\omega](z)=\frac{1}{\Gamma(1-\lambda)} \frac{d}{d z}\left(\int_{0}^{z} \frac{\omega(t)}{(z-t)^{\lambda}} d t\right) \quad(0 \leq \lambda<1),
$$

where the multiplicity of $(z-t)^{-\lambda}$ above is removed requiring $\log (z-t)$ to be real when $z-t>0$. All right, here and throughout this paper, the function $\Gamma$ is the well-known classical gamma function.

Under favour of the definition in (1), for an analytic function $\omega$, the well-known derivative operator, which is the Srivastava-Owa operator of order $v+\lambda$, is then defined by

$$
\mathrm{D}_{z}^{v+\lambda}[\omega](z)=\frac{d^{v}}{d z^{v}}\left(\mathrm{D}_{z}^{\lambda}[\omega](z)\right) \quad(0 \leq \lambda<1 ; v \in \mathbb{N} \cup\{0\}),
$$

which readily yields

$$
\mathrm{D}_{z}^{0+\lambda}[\omega](z)=\mathrm{D}_{z}^{\lambda}[\omega](z) \quad \text { and } \quad \mathcal{D}_{z}^{1+\lambda}[\omega](z)=\frac{d}{d z}\left(\mathrm{D}_{z}^{\lambda}[\omega](z)\right)
$$


where $0 \leq \lambda<1$.

By means of the Srivastava-Owa derivative (see [11]) and fractional calculus operator (see [3], [5]-[7]), several (elementary) operators were also introduced and then presented various results relating with derivative operator of fractional order. One of them also is the Tremblay Operator $\mathrm{T}_{\tau, \lambda}[\cdot]$, which was defined in the domain of the complex plane and whose properties in several spaces were discussed systematically, where

$$
0<\lambda \leq 1, \quad 0<\tau \leq 1 \text { and } 0 \leq \tau-\lambda<1 .
$$

For both some of the details of fractional calculus and some applications identified by certain (elementary) operators (in relation with fractional calculus), one may refer to the scientific investigations given by [1], [6] and [10]-[12].

For an analytic function $g:=g(z)$ belonging to the class $\mathrm{A}(n)$, in general, the Tremblay operator:

$$
\mathrm{T}_{\tau, \lambda}[g](z) \quad \text { or } \quad \mathrm{T}_{\tau, \lambda}[g(z)]
$$

is denoted and

$$
\mathrm{T}_{\tau, \lambda}[g](z):=\frac{\Gamma(\lambda)}{\Gamma(\tau)} z^{1-\lambda} \mathrm{D}_{z}^{\tau-\lambda}\left[z^{\tau-1} g(z)\right],
$$

is also defined, where $0<\tau \leq 1,0<\lambda \leq 1,0 \leq \tau-\lambda<1$ and $z \in \mathbb{U}$.

It can be easily seen that the mentioned operator:

$$
\mathrm{D}_{z}^{\tau-\lambda}[\cdot] \quad(0<\lambda \leq 1 ; 0<\tau \leq 1 ; 0 \leq \tau-\lambda<1)
$$

is also equivalent to the well-known operator, which is called as the Srivastava-Owa operator of fractional derivative of order $\tau-\lambda$ represented by (1).

The following results are only two of various applications of the mentioned operator, defined by (2), will be required for both stating and proving of our basic results.

Lemma 1. ([3]) Let $g(z)$ be in the class $\mathrm{A}(n)$ and also let $0<\tau \leq 1,0<\lambda \leq 1$, and $0 \leq \tau-\lambda<1$. Then,

$$
\mathrm{T}_{\tau, \lambda}[g](z)=\frac{\tau}{\lambda} z+\frac{\Gamma(\lambda)}{\Gamma(\tau)} \sum_{j=n+1}^{\infty} \frac{\Gamma(j+\tau)}{\Gamma(j+\lambda)} a_{j} z^{j} \quad(z \in \mathbb{U}) .
$$

Lemma 2. ([3]) Let $g(z)$ be in the class $\mathcal{A}(n)$ and also let $0<\tau \leq 1,0<\lambda \leq 1$, and $0 \leq \tau-\lambda<1$. Then,

$$
\frac{d}{d z}\left(\mathrm{~T}_{\tau, \lambda}[g](z)\right)=\frac{\tau}{\lambda}+\frac{\Gamma(\lambda)}{\Gamma(\tau)} \sum_{j=n+1}^{\infty} \frac{j \Gamma(j+\tau)}{\Gamma(j+\lambda)} a_{j} z^{j-1} \quad(z \in \mathbb{U}) .
$$

For a complex valued function $g:=g(z)$ belonging to the class $\mathrm{A}(n)$ and admissible values of the parameters $\lambda$ and $\tau$, it is obvious that

$$
z^{-1} \mathrm{~T}_{\tau, \lambda}[g](z) \in \mathrm{H}_{\tau / \lambda}(n)
$$


and

$$
\frac{d}{d z}\left(\mathrm{~T}_{\tau, \lambda}[g](z)\right) \in \mathrm{H}_{\tau / \lambda}(n)
$$

and, specially,

$$
\begin{gathered}
\mathrm{T}_{1,1}[g](z)=g(z) \in \mathrm{A}(n), \\
\frac{d}{d z}\left(\mathrm{~T}_{1,1}[g](z)\right)=g^{\prime}(z) \in \mathrm{H}(n)
\end{gathered}
$$

and also

$$
z^{-1} \mathrm{~T}_{1,1}[g](z)=z^{-1} g(z) \in \mathrm{H}(n),
$$

where $z \in \mathbb{U}$.

\section{A lemma and a comprehensive result}

Both by stating of our main results and for convenience, there is a need to define an operator. In consideration of Eq. (3) (of Lemma 1) and Eq. (4) (of Lemma 2), we now denote it by the following notation:

$$
\mathbb{T}_{g}(z):=\mathbb{T}_{\tau, \lambda}^{\mu}[g](z) \quad(g \in \mathrm{A}(n) ; z \in \mathbb{U})
$$

and also define in the following form:

$$
\mathbb{T}_{g}(z):=\mu \mathrm{T}_{\tau, \lambda}[g](z)+(1-\mu) z \frac{d}{d z}\left(\mathrm{~T}_{\tau, \lambda}[g](z)\right),
$$

where $0 \leq \mu \leq 1, g \in \mathrm{A}(n)$ and $z \in \mathbb{U}$.

As an equivalent form of the operator $\mathbb{T}_{g}(z)$ given by (11), one uses the assertions given in (3) and (4), the following:

$$
\mathbb{T}_{g}(z):=\frac{\tau}{\lambda}\left(z+\sum_{j=n+1}^{\infty}[j(1-\mu)+\mu] \frac{\lambda \Gamma(j+\tau) \Gamma(\lambda)}{\tau \Gamma(k+\lambda) \Gamma(\tau)} a_{j} z^{j}\right)
$$

can be easily determined, where

$$
0 \leq \mu \leq 1,0<\lambda \leq 1,0<\tau \leq 1,0 \leq \tau-\lambda<1 \text { and } z \in \mathbb{U} .
$$

It is obvious that

$$
\text { (14) } \frac{\lambda}{\tau} \mathbb{T}_{g}(z) \in \mathrm{A}(n) \quad, \quad z^{-1} \mathbb{T}_{g}(z) \in \mathrm{H}_{\tau / \lambda}(n) \quad \text { and } \quad \frac{d}{d z}\left(\mathbb{T}_{g}(z)\right) \in \mathrm{H}_{\tau / \lambda}(n) \text {. }
$$


It is also noted that, in the statements like those involved in (5), (9), (15) and similar type rational functions, and in analogous situations throughout in this investigation, it should be understood that functions there, which have removable singularities at the point $z=0$, have had those singularities removed.

As it is indicated in the abstract of this investigation, in this chapter, the operator stated in (11) (or in (12)) will be considered by making use of the help of the mentioned operator defined by (2), a comprehensive result, which also consists of various (analytic and) geometric properties will be constituted. For its proof, the earlier assertion in [8] (along with the (similar) consideration used in [7] (or in [6])), will be allowed there. For this goal, before proving it, the accentuated assertion will be also needed, which is given by the following Lemma (below).

Lemma 3. ([8]) Let

$$
b \geq 1 \quad, \quad a \in \mathbb{R}-\{0\} \quad, \quad p(z) \in \mathrm{H}(n) \quad \text { and } \quad z \in \mathbb{U} .
$$

In that time, if there exists a point $z_{0}$ in the disc $\mathbb{U}$ such that

$$
\Re e(p(z))>0 \quad\left(|z|<\left|z_{0}\right|<1\right) \quad, \quad \Re e\left(p\left(z_{0}\right)\right)=0 \quad \text { and } \quad p\left(z_{0}\right) \neq 0
$$

then

$$
p\left(z_{0}\right)=i a \quad \text { and } \quad z_{0} p^{\prime}\left(z_{0}\right)=i \frac{b}{2}\left(a+\frac{1}{a}\right) p\left(z_{0}\right) .
$$

Now we can state and then prove our main result consisting of various special results.

Theorem 1. Let $g:=g(z) \in \mathrm{A}(n)$ and also let $\mathbb{T}_{g}(z)$ be of the form in (13). For the admissible values of the parameters mentioned in (13), if any one of the cases given in

$$
\Re e\left\{\frac{z \frac{d^{2}}{d z^{2}}\left(\mathbb{T}_{g}(z)\right)}{\frac{d}{d z}\left(\mathbb{T}_{g}(z)\right)}\right\}>\left\{\begin{array}{cll}
\frac{\alpha}{2(\alpha-1)} & \text { when } & 0 \leq \alpha \leq \frac{1}{2} \\
\frac{\alpha-1}{2 \alpha} & \text { when } & \frac{1}{2} \leq \alpha<1
\end{array}\right.
$$

is satisfied, then

$$
\Re e\left\{\frac{d}{d z}\left(\mathbb{T}_{g}(z)\right)\right\}>\frac{\tau}{\lambda} \alpha \quad(0 \leq \alpha<1 ; g \in \mathrm{A}(n) ; z \in \mathbb{U})
$$

is also satisfied.

Proof. Let $g \in \mathrm{A}(n)$ and also let $\mathbb{T}_{g}(z)$ be of the form given by (12). Under the conditions given in (13) and with the help of the definition of the related operator, we now consider a function $p(z)$ as given in the form:

$$
p(z)=\frac{1}{1-\alpha}\left[\frac{\lambda}{\tau} \frac{d}{d z}\left(\mathbb{T}_{g}(z)\right)-\alpha\right]
$$


where $0 \leq \alpha<1, z \in \mathbb{U}$ and $g \in \mathrm{A}(n)$.

Clearly, $p(z)$ is in the class $\mathrm{H}(n)$, namely, it is an analytic function in $\mathbb{U}$ and also satisfies the condition $p(0)=1$. By means of both (3) of Lemma 1 and (4) of Lemma 2 , it follows from (19) that

$$
\frac{z \frac{d^{2}}{d z^{2}}\left(\mathbb{T}_{g}(z)\right)}{\frac{d}{d z}\left(\mathbb{T}_{g}(z)\right)}=\frac{(1-\alpha) z p^{\prime}(z)}{\alpha+(1-\alpha) p(z)} \quad(z \in \mathbb{U}) .
$$

Systematically, in the same logic earlier considered as in [6] and [7], we now suppose that there exists a point $z_{0}$ satisfying the condition:

$$
\Re e\left(p\left(z_{0}\right)\right)=0,
$$

given by (15) in Lemma 3 , where $z_{0} \in \mathbb{U}$ and $p\left(z_{0}\right) \neq 0$. Then, by applying of the assertions in Lemma 3 (in (16)), which are

$$
\Re e\left(p\left(z_{0}\right)\right)=i a \quad \text { and }\left.\quad \frac{z p^{\prime}(z)}{p(z)}\right|_{z=z_{0}}=i \frac{b}{2}\left(a+\frac{1}{a}\right) \quad(a \neq 0 ; \quad b \geq 1),
$$

to the equation given by (20), the following result:

$$
\begin{aligned}
\Re e\left(\left.\frac{z \frac{d^{2}}{d z^{2}}\left(\mathbb{T}_{g}(z)\right)}{\frac{d}{d z}\left(\mathbb{T}_{f}(z)\right)}\right|_{z:=z_{0}}\right) & =\Re e\left(\left.\frac{(1-\alpha) z p^{\prime}(z)}{\alpha+(1-\alpha) p(z)}\right|_{z:=z_{0}}\right) \\
& =-\frac{b}{2} \frac{\alpha(1-\alpha)\left(1+a^{2}\right)}{\alpha^{2}+(1-\alpha)^{2} a^{2}}
\end{aligned}
$$

is then obtained. By taking into account the result given in (21) along with the following inequalities:

$$
\frac{1+a^{2}}{a^{2}+\left(\frac{\alpha}{1-\alpha}\right)^{2}} \geq 1 \text { when } 0 \leq \alpha \leq \frac{1}{2}
$$

and

$$
\frac{1+a^{2}}{1+\left(\frac{1-\alpha}{\alpha}\right)^{2} a^{2}} \geq 1 \text { when } \frac{1}{2} \leq \alpha<1
$$

the following relations:

$$
\Re e\left(\left.\frac{z \frac{d^{2}}{d z^{2}}\left(\mathbb{T}_{g}(z)\right)}{\frac{d}{d z}\left(\mathbb{T}_{g}(z)\right)}\right|_{z:=z_{0}}\right)=-\frac{b}{2} \frac{\alpha(1-\alpha)\left(1+a^{2}\right)}{\alpha^{2}+(1-\alpha)^{2} a^{2}}
$$




$$
\leq\left\{\begin{array}{ccc}
\frac{\alpha}{2(\alpha-1)} & \text { if } & 0 \leq \alpha \leq \frac{1}{2} \\
\frac{\alpha-1}{2 \alpha} & \text { if } & \frac{1}{2} \leq \alpha<1
\end{array}\right.
$$

are easily acquired. But, this result is a contradiction with the result given by (17) of Theorem 1. This means that there is no any point $z_{0}$ satisfying the assumed condition, which is the inequality:

$$
\Re e\left(p\left(z_{0}\right)\right)=0 \quad\left(z_{0} \in \mathbb{U}\right) .
$$

In the present case, it should be in the form:

$$
\Re e(p(z))>0 \quad(\forall z \in \mathbb{U}) .
$$

So, the statement, which is given by the equation (19), then requires that the inequality:

$$
\Re e(p(z))=\Re e\left(\frac{1}{1-\alpha}\left[\frac{\lambda}{\tau} \frac{d}{d z}\left(\mathbb{T}_{f}(z)\right)-\alpha\right]\right)>0 \quad(z \in \mathbb{U}),
$$

which immediately yields the inequality that is that is the provision of Theorem 1 given as (18). Thereby, the desired proof has been completed .

\section{Concluding remarks}

In the last part of this investigation, there are certain conclusions and recommendations. We want now to inform about some of them. As it is indicated, in the first section of this investigation, certain basic information relating to fractional calculus and the Tremblay operator have been first introduced. In the second section, an extensive result composed by the signified operator has been also constituted (and then proven). Specially, we think that, for related researchers, it will also be useful to center on the results earlier present by $[6,7]$ in the references.

With a simple observation, if the parameters in the fractional calculations given in the first section are appropriately chosen, all right, it is possible that many special results can be then generated (or, redetermined). In particular, some special results of those, in relation with analytic and geometric function theory, have been also given in (5)-(9) and (11)-(14). Naturally, those (possible) special cases together with the extensive result will help us to reveal a number of new (or, earlier) specific results. Of course, it is not possible to specify all of them. Nevertheless, we want to present only three of its special implications.

As the first implication, by taking $\mu:=1$ in Theorem 1 , the following-special relation:

$$
\mathbb{T}_{g}(z)\left(:=\mathbb{T}_{\tau, \lambda}^{1}[g](z)\right) \equiv \mathrm{T}_{\tau, \lambda}[g](z)
$$


is easily shown up, which also is the assertion given by Lemma 1 when, of course, $g:=g(z) \in \mathrm{A}(n)$. In the present case, the foreknown result (see Theorem 1, p. 299 in [7]) can be then represented, which is the following implication (below).

Implication 1. Let $g:=g(z) \in \mathrm{A}(n)$ and also let $\mathrm{T}_{\tau, \mu}[g](z)$ be in the form (3). For the admissible values of the parameters $\tau$ and $\lambda$, indicated in Lemma 1, if any one of the cases contained in

$$
\Re e\left\{\frac{z \frac{d^{2}}{d z^{2}}\left(\mathrm{~T}_{\tau, \lambda}[g](z)\right)}{\frac{d}{d z}\left(\mathrm{~T}_{\tau, \lambda}[g](z)\right)}\right\}>\left\{\begin{array}{cl}
\frac{\alpha}{2(\alpha-1)} & \text { when } 0 \leq \alpha \leq \frac{1}{2} \\
\frac{\alpha-1}{2 \alpha} & \text { when } \frac{1}{2} \leq \alpha<1
\end{array}\right.
$$

holds, then

$$
\Re e\left\{\frac{d}{d z}\left(\mathrm{~T}_{\tau, \lambda}[g](z)\right)\right\}>\frac{\tau}{\lambda} \alpha
$$

also holds, where $0 \leq \alpha<1, g \in \mathrm{A}(n)$ and $z \in \mathbb{U}$.

As the second implication, by setting $\mu:=0$ in Theorem 1 , the following equivalent relation:

$$
\mathbb{T}_{g}(z)\left(:=\mathbb{T}_{\tau, \lambda}^{0}[g](z)\right) \equiv z \frac{d}{d z}\left(\mathrm{~T}_{\tau, \lambda}[g](z)\right)
$$

is then deduced, which also is the assertion given by Lemma 2 when, of course, $g:=g(z) \in \mathrm{A}(n)$. In that case, the special result can be then reestablished, which is Implication 2.

Implication 2. Let $g:=g(z) \in \mathrm{A}(n)$ and also let $\mathrm{T}_{\tau, \lambda}[g](z)$ be in the form (3). For the admissible values of the parameters $\tau$ and $\lambda$, signified by Lemma 2, if any one of the cases presented in

$$
\begin{aligned}
\Re e\left\{\frac{2 z \frac{d^{2}}{d z^{2}}\left(\mathrm{~T}_{\tau, \lambda}[g](z)\right)+z^{2} \frac{d^{3}}{d z^{3}}\left(\mathrm{~T}_{\tau, \lambda}[g](z)\right)}{\frac{d}{d z}\left(\mathrm{~T}_{\tau, \lambda}[g](z)\right)+z \frac{d^{2}}{d z^{2}}\left(\mathrm{~T}_{\tau, \lambda}[g](z)\right)}\right\} \\
>\left\{\begin{array}{cl}
\frac{\alpha}{2(\alpha-1)} & \text { when } 0 \leq \alpha \leq \frac{1}{2} \\
\frac{\alpha-1}{2 \alpha} & \text { when } \frac{1}{2} \leq \alpha<1
\end{array}\right.
\end{aligned}
$$

is true, then

$$
\Re e\left\{\frac{d}{d z}\left(\mathrm{~T}_{\tau, \lambda}[g](z)\right)+z \frac{d^{2}}{d z^{2}}\left(\mathrm{~T}_{\tau, \lambda}[g](z)\right)\right\}>\frac{\tau}{\lambda} \alpha
$$

is also true, where $0 \leq \alpha<1, g \in \mathrm{A}(n)$ and $z \in \mathbb{U}$.

As the last implication, by letting $\lambda:=\tau:=1$ in Implication 1 (or, $\mu:=\tau:=$ $\lambda:=1$ in Theorem 1), the following-equivalent relationships:

$$
\mathbb{T}_{g}(z)\left(:=\mathbb{T}_{1,1}^{1}[g](z)\right) \equiv \mathrm{T}_{1,1}[g](z) \equiv g(z)
$$


are then achieved, when $g:=g(z) \in \mathrm{A}(n)$. In this case, the more-special result, which also relates to Geometric Function Theory (see, for its details, [2], [4], [9] and [13]), can be easily revealed, which is the following proposition.

Implication 3. (Proposition 1, p. 303 in [7]) If a function $g \in \mathrm{A}(n)$ satisfies any one of the cases composed in

$$
1+\Re e\left(\frac{z g^{\prime \prime}(z)}{g^{\prime}(z)}\right)>\left\{\begin{array}{ccc}
\frac{2-3 \alpha}{2(1-\alpha)} & \text { when } \quad 0 \leq \alpha \leq \frac{1}{2} \\
\frac{3 \alpha-1}{2 \alpha} & \text { when } \quad \frac{1}{2} \leq \alpha<1
\end{array},\right.
$$

then

$$
\Re e\left(g^{\prime}(z)\right)>\alpha \quad(0 \leq \alpha<1 ; z \in \mathbb{U}),
$$

that is to say that the function $g(z)$ is a close-to-convex of order $\alpha$ in $\mathbb{U}$.

\section{References}

[1] M. P. Chen, H. Irmak, H. M. Srivastava, A certain subclass of analytic functions involving operators of fractional calculus, Comput. Math. Appl., vol. 35, no. 2, 1998, 83-91.

[2] P. L. Duren, Grundlehren der Mathematischen Wissenchaffen, Springer-Verlag, New York, Berlin, Heidelberg, Tokyo, 1983.

[3] Z. Esa, H. M. Srivastava, A. Kılıçman, R. W. Ibrahim, A novel subclass of analytic $f$ unctions specified by a family of fractional derivatives in the complex domain, Filomat, vol. 31, no. 9, 2017, 2837-2849.

[4] A. W. Goodman, Univalent Functions, Mariner Publishing Co., Inc., Tampa, FL, vol. I, II, 1983.

[5] R. W. Ibrahim, J. M. Jahangiri, Boundary fractional differential equation in a complex domain, Boundary Value Prob., Article ID 66, 2014, 1-11.

[6] H. Irmak, Certain basic information related to the Tremblay operator and some applications in connection therewith, Gen. Math., vol. 27, no. 2, 2019, 13-21.

[7] H. Irmak, O. Engel, Some results concerning the Tremblay operator and some of its applications to certain analytic functions, Acta Univ. Sapientiae Math., vol. 11, no. 2, 2019, 296-305.

[8] M. Nunokawa, On properties of non-Caratheodory functions, Proc. Japan Acad. Ser. A Math. Sci., vol. 68, no. 6, 1992, 152-153.

[9] Z. Nehari, Conformal mapping, McGraw-Hill Book Company, New York, Toronto and London, 1952. 
[10] S. Owa, On the distortion theorems. I, Kyungpook Math. J., vol. 8, no. 1, 1978, 53-59

[11] H. M. Srivastava, S. Owa (Editors), Univalent functions, fractional calculus and their applications, Halsted Press, John Wiley and Sons, New York, Chieschester, Brisbane, Toronto, 1989.

[12] M. P. Chen, H. M. Srivastava, C. S. Yu, Some operators of fractional calculus and their applications involving a novel class of analytic functions, Appl. Math. Comput., vol. 91, no. 1-2, 1998, 285-296.

[13] C. Pommerenke, Univalent Functions, Vandenhoeck \& Ruprecht, Göttingen, 1975.

Hüseyin Irmak

Çankırı Karatekin University

Faculty of Sciences

Department of Mathematics

Uluyazı Campus

TR 18100, Çankırı, Turkey

e-mail: hirmak@karatekin or hisimya@yahoo.com 